\title{
Noncircular iris segmentation based on weighted adaptive hough transform using smartphone database
}

\begin{abstract}
Iris segmentation methods work based on ideal imaging conditions which produce good output results. However, the segmentation accuracy of an iris recognition system significantly influences its performance, especially with data that captured in unconstrained environment of the Smartphone. This paper proposes a novel segmentation method for unconstrained environment of the Smartphone videos based on choose the best frames from the videos and try to enhance the contrast of this frames by applying the two fuzzy logic membership functions on the negative image which delimit between dark and bright regions in able to make the dark region darker and the bright region brighter. This pre-processing step Facilitates the work of the Weighted Adaptive Hough Transform to automatically find the diameter of the iris region to apply the osiris v4.1. The proposed method results on the video of (Mobile Iris Challenge Evaluation (MICHE))-I, iris databases indicate a high level of accuracy and more efficient computationally using the proposed technique.
\end{abstract}

Keyword: Biometrics; Fuzzy logic membership function; Hough Transform; Iris segmentation methods; Miche-I; Smartphone iris; Weighted adaptive hough transform 